\title{
FUTURE OF HEALTH POLICY \& SYSTEMS RESEARCH: TRANSITIONING FROM MILLENNIUM DEVELOPMENTAL GOALS TO SUSTAINABLE DEVELOPMENTAL GOALS FOR IMPROVING HEALTH
}

\author{
Anam Feroz ${ }^{1}$, Imran Naeem Abbasi ${ }^{2}$, Wafa Aftab ${ }^{2}$ and Fauziah Rabbani ${ }^{3}$
}

\author{
${ }^{1}$ Research Fellow at the Department of Community Health Sciences, Aga Khan University, PO Box 3500, Stadium \\ Road, Karachi, Pakistan \\ ${ }^{2}$ Senior Instructor at the Department of Community Health Sciences, Aga Khan University, PO Box 3500, Stadium \\ Road, Karachi, Pakistan. \\ ${ }^{3}$ Associate Vice Provost \& Professor and Chair Dept. of Community Health Sciences at the Aga Khan University, PO \\ Box 3500, Stadium Road, Karachi, Pakistan. \\ Correspondence: Fauziah Rabbani. Email: fauziah.rabbani@aku.edu
}

\begin{abstract}
\section{Summary}

The importance of health policy and systems research (HPSR) for strengthening health systems is widely recognized. However, availability and uptake of research evidence for health systems to formulate evidenceinformed policies is challenging. The transition from Millennium Development Goals to Sustainable Development Goals (SDG) represents a new global promise to health by encompassing a broader range of economic, environmental, and social pillars of sustainable development. The adoption of the 2030 agenda offers an exciting opportunity for strengthening health system governance. The integration of HPSR is essential for the fulfillment of SDGs through the collaboration of multiple sectors and policy coherence. Various innovative strategies have been recognized to build strong partnerships between researchers, policy-makers, and academicians to highlight the role of HPSR in realizing and achieving SDGs. Multilateral and bilateral organizations working in the field of HPSR can play a facilitatory role to inform the development of healthcare reforms through strategic engagement among a variety of stakeholders.
\end{abstract}

Keywords: Millennium development goals, sustainable developmental goals, transitioning from mdgs to sdgs, health policy and systems research, research to policy, health systems strengthening

Role of Health Policy and Systems Research in Strengthening Health Systems

The importance of Health policy and systems research (HPSR) has been recognized for a long time by World Health Organization (WHO) and other bodies as a tool to inform policy-making and planning for health service delivery $(1,2)$. However, availability of research evidence as per needs of the health systems is challenging and even more so is the uptake of research findings by policy makers to formulate evidenceinformed policies (3). Historically, HPSR has played a pivotal role in strengthening national health programs and service delivery. In Indonesia, findings from a research project led to development of more effective and less costly vector control schedules in malaria control programmes (4). Similarly in Malaysia, use of research findings led to reduced waiting time in hospitals which consequently brought improvement in service delivery (4). In Egypt, health ministry as part of urban health systems development project undertook a research study in order to understand the provider and client side issues in maternal and child health services. Findings of the study shed deeper insight into the services and were used by the health ministry to improve health services (5).

Attempts have been made in Pakistan to conduct research in ways that can be best linked to the national programs and to improve the uptake of research findings by policy makers. One such example is of HIVIAIDS and depicts how researchers made efforts to influence the policy of the National Aids Control Program (NACP). One of the key strategies adopted was creating a strong link with the leadership of NACP, civil society, and the community as key stakeholders in shaping the direction of the research. Researchers were optimistic about the influence of research findings via interactions during various activities with the policy makers (6). Moreover, research on Cotrimoxazole proved that it is effective in 
treating less severe childhood pneumonia leading to the subsequent revision of WHO's guidelines(7).

Transitioning from MDGs to SDGs \& Challenges in the Context of 2030 Agenda

All the research to system strengthening efforts were achieved within the broader framework of Millennium Development Goals (MDGs). However, MDGs signified a comparatively limited scope of human development indicators, among which health-related goals were prominent. The Sustainable Development Goals (SDGs), in contrary, now represent a broader range of economic, environmental, and social pillars of sustainable development (8). SDG 3 on health -"Ensure healthy life and promote well-being for all at all ages"- is one amongst the 17 SDGs. This SDG on health sets out the target in a manner that differentiates between the ones that are carried on and expanded from the MDGs, those that are newly added, and the ones that are indicated as specific "means of implementation"(8).

This transition from MDGs to SDGs is momentous and reflects in many ways a better understanding of development. While MDGs were developed mainly by the countries and organizations of the global North, SDGs are the result of comprehensive global consultation and acknowledge the need to include the global southern stakeholders with a nod to lessons learned from MDGs (8).

SDGs also, represent, to an extent, both how the concept of development has changed in the last 20 years as well as how new developmental problems have arisen. Resultantly, SDGs frame many social and development problems as intersectional issues rather than problems in silos. From a purely health outcomes perspective, 'sustainable' achievement of health goals is thus correctly embedded in a program of achievement of broader determinants of health and well-being alongside focused health goals(8). While this comprehensiveness makes SDGs seem more in tune with the true issues of development; it also poses problems of implementation. Transforming this insight into concrete actions is one of the crucial challenges of the 2030 agenda for sustainable development.

Key Strategies to Promote the Use of HPSR in 2030 Agenda for Sustainable Development

The adoption of the SDGs offers an exciting opportunity for strengthening health system governance through the use of policy developments at international, regional, and country level. The SDG 2030 agenda would require that the emphasis on global health be centered to a larger set of local priorities in health and other disciplines. This would also require optimum allocation of scarce resources for health system governance and cross-sectoral effort which is implicit in the new SDGs 2030 agenda(8).

Another distinguishing feature of SDGs from MDGs is the envisioned involvement of private sector. There is a strong case for private sector involvement in SDG implementation. This applies to health goals as well as their broader determinants. In many developing countries private providers and civil society organizations (CSOs) deliver health services particularly in disadvantaged areas and where public services are weak or inaccessible; involving them in SDG implementation is crucial for achieving the goal of "no one left behind"(8).

Having a favorable policy environment is also immensely important for conducting policy-relevant research and its successful integration into the national policy. One such trial from Mwanza, Tanzania demonstrates that personal links between researchers and policymakers and a conducive policy environment were the key factors leading to a policy shift following results of the trial demonstrating that improved treatment services for sexually transmitted infections were successful in preventing HIVIAIDS (9).

The MDG experience also provides lessons to tackle some of these hurdles and develop new strategies. This is particularly true for data availability and measurement of progress. When implementation of MDGs began, availability of uniform and reliable data sources that could be used to provide baseline data was limited. Considerable work has gone into creating, updating and harmonizing data sources. Given the experience of MDGs, it would be better to utilize already existing data sources to minimize resource needs instead of conducting large surveys and creating SDG-specific data (10).

Another strategy is linking Essential Public Health Functions (EPHFs) viz strengthening national programs, health care quality assurance and improving the effectiveness of health programs etc. with HPSR and SDGs. One of the key EPHF is advancing public health research in order to inform and influence the health policy and the practice. Considering the scope of HPSR, therefore, it is not hard to imagine its critical role in improving the EPHFs in particular and how HPSR can strengthen health systems and contribute to attaining health-related SDGs in general $(8,11)$.

The integration of HPSR is essential to frame SDGs in such a way that their fulfillment requires the collaboration of multiple sectors and policy coherence. With improved recognition of the significant role that HPSR has to play in accomplishing SDGs, there is a high need to develop fundamental curricula on HPSR in postgraduate courses, organize scientific meetings to build interdisciplinary competencies among health policy researchers, conduct methodological dialogue and provide exchange opportunities in academic institutions(12).

Case studies from China, India, Mexico, and Thailand significantly explain the use of HPSR in convincing healthcare reforms to build stronger health systems. The common factors in their success include establishing HPSR institutions in universities and in independent research institutes; involving legendary health researchers in advocating the role and promise of 
HPSR; increasing provision of resources for HPSR; allowing public opinion in highlighting important concerns related to HPSR; recognizing the involvement of global funding agencies in strengthening the generation of evidence-base; and developing stronger HPSR linkages at the local, regional and global level (12). All of these innovative strategies call for strong partnerships between researchers and policy makers and academicians.

Opportunities to Further Strengthen the Role of Large Multilateral and Bilateral Organizations

Large multilateral and bilateral organizations can advise HPSR stakeholders and can direct future strategic investments for building HPSR capacity while addressing some of the key national HPSR priorities. Organizations working in the field of HPSR, including Alliance for Health Policy and Systems Research (AHPSR) can be helpful in both engaging policy makers, academicians, and researchers so that SDG-related research findings are implemented expeditiously and also in creating a long-term culture of research-policy collaboration(12).

Such organizations can best be utilized in a facilitatory role in order to conceptualize at various levels, based predominantly on their policy research expertise and global, regional and country-level connections with policy makers and research community. At the international level, these organizations can help shape the broader SDG research agenda. At the regional level, such organizations can play a pivotal role in harmonizing various research activities to avoid needless duplication of research efforts. At the country level, such facilitation can help knowledge sharing and translation of identified global and regional research needs into policy research priorities.

Such organizations can play a leading role in organizing scientific meetings to provide opportunities for methodological dialogue among multidisciplinary constituencies. These organizations can support countries in strengthening health systems through building the foundations of HPSR in under-developed and developing states to help achieve the goal of Universal health coverage (UHC).

These organizations can serve as agents of change to capitalize on the unique potential of HPSR and to inform the development of healthcare reforms. The organizations should be facilitatory in overcoming some of the main national HPSR challenges, which include weak HPSR organizational and individual capacity, lack of partnerships between various disciplines and constituencies, weak evidenced-informed policy processes, and lack of national HPSR priority setting. Lastly, the organizations can support and enable health system planners and administrators to make decisions that are informed by research through creating knowledge resources, issuing specific guidance to planners, and hosting consultations on policy uptake.

Word count (Including references and summary):
2,015

\section{References}

1. Alliance for Health Policy and Systems Research. What is Health Policy and Systems Research (HPSR)? 2016.

2. Hanney SR, Gonzalez-Block MA, Buxton MJ, Kogan M. The utilisation of health research in policy-making: concepts, examples and methods of assessment. Health research policy and systems. 2003;1(1):2.

3. Waddell C, Lavis JN, Abelson J, Lomas J, Shepherd CA, Bird-Gayson T, et al. Research use in children's mental health policy in Canada: Maintaining vigilance amid ambiguity. Social science \& medicine. 2005;61(8):1649-57.

4. Mills A. Strengthening health systems: the role and promise of policy and systems research: World health organization (WHO). Alliance for health policy and systems research; 2004.

5. World Health Organization. Health systems research in action: case studies from Botswana, Colombia, Egypt, Indonesia, Malaysia, the Netherlands, Norway and the United States of America. 1988.

6. Hawkes S, Zaheer HA, Tawil O, O'Dwyer M, Buse K. Managing research evidence to inform action: Influencing HIV policy to protect marginalised populations in Pakistan. Global public health. 2012;7(5):482-94.

7. Straus WL, Qazi SA, Kundi Z, Nomani NK, Schwartz B, Group PC-tS. Antimicrobial resistance and clinical effectiveness of cotrimoxazole versus amoxycillin for pneumonia among children in Pakistan: randomised controlled trial. The Lancet. 1998;352(9124):270-4.

8. Boerma T, Mathers C, AbouZahr C, Chatterji S, Hogan D, Stevens G, et al. Health in 2015: From MDGs Millennium Development Goals to SDGs Sustainable Development Goals.

9. Philpott A, Maher D, Grosskurth H. Translating HIVIAIDS research findings into policy: lessons from a case study of 'the Mwanza trial'. Health Policy and Planning. 2002;17(2):196-201.

10. United Nations. Data Challenges and Solutions for the SDG's 2015.

11. World Health Organization. Strengthening essential public health functions in support of the achievement of universal health coverage. 2016.

12. World Health Organization. Changing mindsets: strategy on health policy and systems research. Geneva:WHO. 2012. 